\title{
Téoros
}

Revue de recherche en tourisme

\section{Entretien avec Irène dos Santos}

\section{Marie-Blanche Fourcade}

Volume 29, numéro 1, 2010

URI : https://id.erudit.org/iderudit/1024766ar

DOI : https://doi.org/10.7202/1024766ar

Aller au sommaire du numéro

\section{Éditeur(s)}

Université du Québec à Montréal

\section{ISSN}

0712-8657 (imprimé)

1923-2705 (numérique)

Découvrir la revue

\section{Citer ce document}

Fourcade, M.-B. (2010). Entretien avec Irène dos Santos. Téoros, 29(1), 130-131. https://doi.org/10.7202/1024766ar d'utilisation que vous pouvez consulter en ligne.

https://apropos.erudit.org/fr/usagers/politique-dutilisation/ 


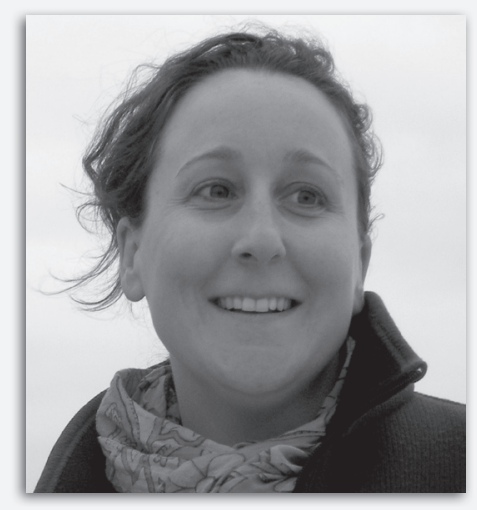

Propos recueillis par Marie-Blanche Fourcade, rédactrice invitée

Irène dos Santos est ethnologue. Elle vient de soutenir sa thèse de doctorat en anthropologie à l'École des hautes études en sciences sociales de Paris, intitulée Les brumes de la mémoire : expérience migratoire et quête identitaire de descendants de migrants portugais de France. Dans le cadre de son doctorat, elle a été boursière de la Fundação para a Ciência e a Tecnologia, rattachée au Laboratoire d'anthropologie sociale (UMR CNRS-EHESS-Collège de France). Ses recherches menées depuis une dizaine d'années en France et au Portugal portent sur la migration portugaise, intra-européenne, et sur les descendants de migrants, en particulier. Elle a, entre autres, animé le séminaire de recherche du Groupe d'anthropologie du Portugal de la Fondation Maison des sciences de l'homme de Paris (2001-2004) et participé à plusieurs projets de films documentaires en lien avec ses recherches.

\section{Quel est le parcours qui vous a menée à réaliser une enquête ethnographique entre la France et le Portugal sur les enfants et petits-enfants de migrants portugais?}

En 1998, dans le cadre d'un mémoire de D.E.A. (diplôme d'études approfondies), j'ai commencé à m'intéresser à la population portugaise et d'origine portugaise, caractérisée comme « invisible » et «bien intégrée » en France. Ma recherche de terrain a porté sur une association portugaise de la région parisienne, créée au début des années 1970 autour de deux activités principales : la danse folklorique et le football, et c'est sur le groupe de danse folklorique que mon travail a plus particulièrement porté. Ce groupe était composé de familles et constituait un espace collectif de la transmission culturelle et de la fidélité à l'« origine». J'ai ensuite poursuivi cette recherche dans la cadre de ma thèse de doctorat, en travaillant sur un autre type d'association créée par des jeunes étudiants et diplômés dans différentes grandes villes et universités françaises. Ces associations ont pour caractéristiques de renvoyer à un seul âge de la vie - des «jeunes" - et de rassembler des individus ayant une mobilité sociale ascendante. Les images d'un Portugal européen, «moderne », touristique, et d'une origine portugaise valorisée en tant que telle, ont remplacé la pratique folklorique, symbole d'un Portugal rural, sous-développé, qui renvoie par ailleurs à l'idéologie de la dictature salazariste (1926-1974), mais cette dimension politique est rarement revendiquée par ces jeunes assez ignorants de l'histoire portugaise contemporaine. Ma recherche montre que l'émancipation des contraintes sociales et culturelles héritées dans une situation de forte domination sociale et culturelle ne passe pas nécessairement par l'oubli de l'origine stigmatisante, mais par un processus complexe de réappropriation de celle-ci. Cette réinvention est rendue possible, dans ce cas précis, par l'intégration dans la société d'installation, mais aussi par des ressources identitaires puisées dans les transformations du pays d'origine et dans la mémoire familiale, toujours ancrée dans le lieu d'origine. Une des conclusions de cette recherche est que les politiques de la mémoire, la dite "mémoire de la migration », occultent en quelque sorte la "mémoire en migration " qui fonde des pratiques migratoires et des liens au pays d'origine vécus au présent et non au passé.

\section{Quelle est la méthode d'enquête que vous avez privilégiée?}

Elle a évolué au cours de ces différentes recherches. D'une monographie portant sur une association ancrée dans une ville de la banlieue parisienne, l'enquête a pris la forme d'une enquête multisite assez complexe, menée à la fois en France et au Portugal. Lors de mon D.E.A., j'avais déjà pris conscience de l'intérêt d'accompagner mes informateurs au Portugal, lors des vacances, afin d'y observer leurs modes de participation aux fêtes de village et aux festivals de folklore, très nombreux dans cette période de retrouvailles entre locaux et migrants. Cette idée de suivre mes informateurs au Portugal s'est rapidement concrétisée dans le cadre d'une deuxième enquête menée dans l'association d'étudiants à Paris, puisque certains de ses membres participaient à des « rencontres de lusodescendants " (rencontres européennes et mondiales) au Portugal. J'ai assisté à ces différentes manifestations avec eux et les ai aussi suivis quand, en dehors de ces rencontres institutionnelles, politisées, médiatisées et urbaines, ils allaient rendre visite à leur famille dans les villages d'origine. Cette première expérience d'ethnographie itinérante s'est ensuite multipliée, puisque j'ai, dès lors, suivi mes informateurs lors des divers séjours au Portugal, pour les vacances, mais aussi pour des projets étudiants, professionnels, ou encore pour la célébration religieuse de leur mariage (alors que le mariage civil avait été célébré en France). J'ai également fait l'inverse : rencontrer des informateurs au Portugal que j'ai suivis en France. II s'agit là d'un cas particulier, celui d'une recherche menée sur un rite d'initiation à l'âge d'homme auquel participent des fils d'émigrants. Finalement, la complexité de l'enquête est aussi liée au fait que ma recherche ne porte pas uniquement sur les associations et sur les descendants impliqués dans celles-ci. J'ai voulu confronter l'expérience migratoire vécue par les jeunes engagés dans des revendications identitaires collectives à celle vécue par des jeunes non impliqués et donc beaucoup moins visibles. 


\section{De quelle manière le projet de retour des migrants s'est imposé dans votre recherche?}

La problématique s'est imposée d'emblée. Ces retours sont très fréquents du fait de la proximité géographique de la France et du Portugal. Ils prennent des formes diverses : individuelles ou familiales, envisagées comme définitives ou temporaires, pour les vacances, pour des projets étudiants ou professionnels. Ils sont liés au projet migratoire familial : un retour sans cesse ajourné ayant profondément marqué les enfants de migrants portugais et, aujourd'hui, le retour des parents au moment de la retraite. Les retours ont lieu dans le village d'origine, où une maison a été construite (l'une des raisons premières de l'émigration), qui est le lieu de mémoire, le lieu d'ancrage généalogique. Cette dernière sert de repère dans le cas d'un retour envisagé comme définitif par les jeunes, en milieu urbain. Ce type de retour a correspondu à une conjoncture économique favorable, au début des années 2000, le Portugal ayant connu un taux de chômage beaucoup plus bas qu'en France. Enfin, à cette même époque, a émergé un discours étatique d'appartenance nationale envers les descendants d'émigrants et certains ont pensé qu'ils avaient davantage leur place dans la société d'origine que dans celle d'accueil.

\section{Comment se sont traduites votre observation participante et votre implication sur le terrain, en France et au Portugal? L'observation participante a été différente à la fois selon l'objet étudié (association, rencontre de lusodescendants, famille) et selon les pays. Par exemple, alors qu'il m'a été très difficile de pénétrer la sphère familiale, de vivre avec les familles en France, au Portugal, cela a été beaucoup plus simple. Cette situation s'explique par l'image que mes interlocuteurs ont du lieu de résidence, celui du Portugal étant souvent le plus valorisé. Mais cela s'explique aussi par le fait que, vivant moi-même à Paris, il semblait moins légitime de m'inviter à leur domicile. En ce qui concerne l'observation participante dans les associations de jeunes et les rencontres de lusodescendants, j'y étais d'emblée légitime du fait de mon origine portugaise, immédiatement perceptible par mon patronyme, de mon âge et de mon statut de célibataire. J'ai participé aux activités des associations en m'impliquant dans l'organisation d'évènements, assurant des permanences pour comprendre leur fonctionnement.}

\section{À propos du positionnement du chercheur, toujours, comment a été accueilli le fait que vous soyez vous-même d'origine portugaise?}

Mon origine portugaise a donc initialement été un atout par la forte identification de mes informateurs et par l'idée du partage d'une expérience commune avec moi qui m'ont évité l'effort souvent nécessaire pour être socialement acceptée par la population étudiée. Mais cette origine commune s'est aussi révélée être un handicap, parce qu'elle rendait la démarche du chercheur plus ambiguë, les raisons de ma présence et de mon implication plus floues.

\section{C'est un terrain très prenant et sur le long terme. Comment se} positionne-t-on en tant que chercheur?

Ce type de terrain "proche», au sens spatial et culturel, demande au chercheur une incessante réflexivité. Par mon origine portugaise, je suis considérée comme étant de fait, "par le sang ", une «lusodescendante», pour reprendre la catégorie utilisée par l'État portugais et certaines associations de jeunes. Cette appartenance rend implicite une allégeance. C'est au niveau collectif, communautaire, que mon positionnement en tant que chercheure a été le plus difficile à défendre. Je ne souhaitais pas être la porte-parole d'un discours identitaire sur lequel je travaillais, comme me le demandaient certains leaders associatifs.

\section{Au terme de cette aventure scientifique, continuez-vous à vous impliquer dans la communauté?}

Je ne peux pas dire que je m'implique dans la communauté portugaise, mais j'ai gardé des liens avec des familles et certains informateurs que je revois volontiers en France et au Portugal. Je souhaite continuer à travailler sur les récits de la migration, à m'impliquer dans des projets qui favorisent le dialogue intergénérationnel. Par contre, je ressens une certaine crainte d'être «récupérée» au niveau communautaire, de me retrouver dans les pages d'un journal national, au Portugal, ou communautaire, en France, présentée comme une «lusodescendante de succès», de devenir une caution, ce qui est déjà arrivé... Cet aspect complique aussi la restitution de la recherche.

\section{Quels sont les leçons ou le bilan que vous tirez autour de l'engagement actif des chercheurs sur le terrain?} Je parlerais plutôt d'observation participante que d'engagement. Celle-ci me semble essentielle pour mener une recherche approfondie. Ce type de recherche requiert beaucoup de temps et de la distance. Du temps parce que, d'un point de vue méthodologique, une recherche multisituée fondée sur des réseaux sociaux plutôt que sur une communauté bilocalisée (originaire d'une même localité au pays d'origine) est difficile à mettre en place. Une analyse des constructions identitaires qui prend en compte les successifs âges de la vie de l'individu prend, évidemment, aussi du temps. Enfin, de la distance vis-à-vis de l'objet d'étude est nécessaire pour comprendre toute la complexité de l'enchevêtrement des identités qui renvoient à des échelles d'analyse multiples (l'individu, la famille, le groupe communautaire, la nation), pour dépasser les identités hégémoniques, mais aussi pour déconstruire les catégories d'analyse, tels «deuxième génération » ou encore «lusodescendant», parfois utilisées par les chercheurs sans questionnement préalable. Aller au-delà du sens commun est particulièrement fondamental lorsque nous travaillons sur des problématiques liées aux phénomènes migratoires.

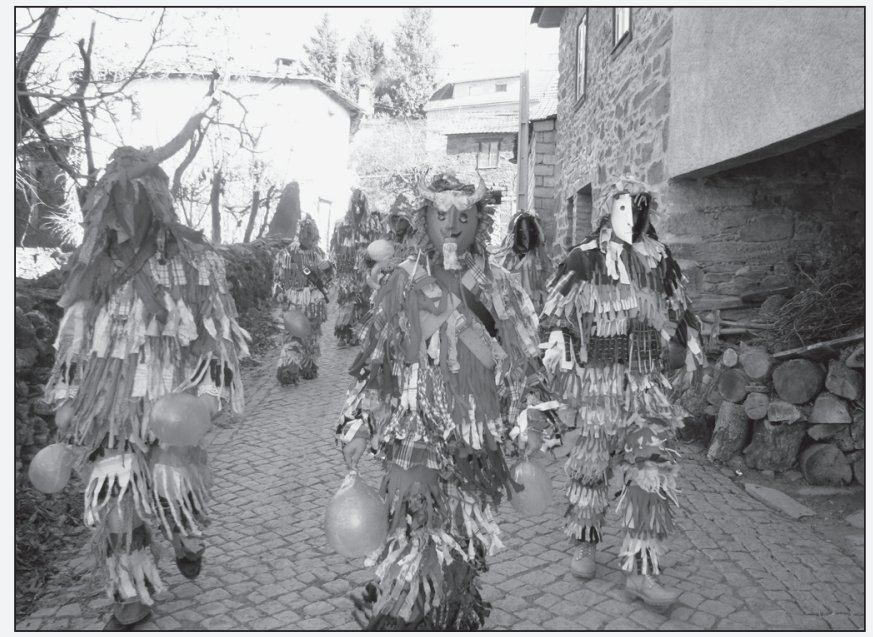

«Fête des garçons » à laquelle participent des fils d'émigrants, décembre 2008, Aveleda (Portugal) (photo : Irène Dos Santos). 